\title{
Uncertain graph generating approach based on differential privacy for preserving link relationship of social networks
}

\section{Jun Yan}

School of Computer Science, Shaanxi Normal University, Xi'an 710119, China

and

School of Mathematics and Computer Applications,

Shangluo College,

Shangluo 726000, China

Email: yanrongjunde@snnu.edu.cn

\section{YuPan Tian}

School of Computer Science, Shaanxi Normal University,

Xi'an 710119, China

Email: YupanTian@snnu.edu.cn

\section{Hai Liu}

Guizhou Provincial Key Laboratory of Public Big Data, Guiyang 550025, China

Email: liuhai@snnu.edu.cn

\section{ZhenQiang $\mathrm{Wu}^{*}$}

School of Computer Science, Shaanxi Normal University, Xi'an 710119, China

Email: zqiangwu@snnu.edu.cn

*Corresponding author

\begin{abstract}
With the widespread use of social networks in our daily life, the personal privacy in social networks has become a growing concern. To prevent the link relationship of social networks from disclosing users' sensitive information when the social networks data is released, an uncertain graph approach based on differential privacy is introduced, which can resist attacks based on background knowledge and possesses better data utility. In this approach, we propose modification of edges based on random response (MERR) algorithm and injection of uncertainty based on $k$-edges-differential privacy (IUDP) algorithm. The MERR algorithm can modify the edge of original graph according to random response mechanism, while the IUDP algorithm injects uncertainty to generate an uncertain graph. For evaluating our approach, the expectation of editing distance between two graphs is adapted to measure the level of privacy preserving. In addition, our approach is conducted in different datasets and compared with other approaches. The experimental results indicate that this approach achieves differential privacy and has better data utility.
\end{abstract}

Keywords: uncertain graph; $k$-edges differential privacy; random response mechanism; Laplace mechanism; link relationship.

Reference to this paper should be made as follows: Yan, J., Tian, Y., Liu, H. and Wu, Z. (2022) 'Uncertain graph generating approach based on differential privacy for preserving link relationship of social networks', Int. J. Security and Networks, Vol. 17, No. 1, pp.28-38.

Biographical notes: Jun Yan received his MS in College of Earth Exploration Science and Technology, Jilin University. He is currently pursuing his PhD in School of Computer Science, Shaanxi Normal University. His research interests include network security and privacy preserving. 
YuPan Tian received her MS in School of Computer Science, Shaanxi Normal University. Her research interests include network security and privacy preserving.

Hai Liu received his BS in 2012 and MS in 2015 from the Guizhou University, and obtained his $\mathrm{PhD}$ in 2019 from the School of Computer Science, Shaanxi Normal University. His main research interest includes privacy preserving.

ZhenQiang Wu received his BS in 1991 from the Shaanxi Normal University, China and MS and $\mathrm{PhD}$ in 2002, and 2007 respectively, all from the Xidian University, China. He is currently a Full Professor of Shaanxi Normal University, China. His research interests include computer communications networks, mainly wireless networks, network security, anonymous communication, and privacy preserving etc. He is a member of ACM and senior of CCF.

\section{Introduction}

With the fast development of social networks, nowadays, online social networks (OSNs) has greatly changed our daily life. For instance, through social networks, people can make friends and share their experiences whenever and wherever. Furthermore, their daily life is closely linked to social networks, such as network payment, online work. However, as a great quantity of users' privacy information has also been retained in OSNs, such as personal account, password and social relationship, it is critical to prevent personal privacy disclosure in social network. For example, Facebook suffered the privacy leakage accident in 2018, in which the information of 50 million Facebook users was illegal used by the Cambridge Analysis Company, and the users' privacy information was applied to predict elections and influence votes (Khandelwal, 2018). Therefore, when social networks data will be published, we must carry out privacy preserving for this data to prevent the data mining from leaking users' privacy information.

Owing to attacks based on the link relationships of social network, many approaches based on graph modification technology have been proposed in Casas-Roma et al. (2016), which provided stronger privacy preserving than the simple anonymity approaches (Backstrom et al., 2007; Zhou and Pei, 2008). Particularly, there are three important categories in graph modification approaches: edge and node modification approaches, generalisation approaches, and uncertain approaches.

In edge and vertex modification approaches, there were random modification approaches and constrained modification approaches. In random modification approaches, considering the important edges in graph, Casas-Roma (2014) proposed an algorithm to maximise data utility while satisfying a desired privacy level, which was better than the approach presented by Hay et al. (2007). Ying et al. (2009) investigated how an edge-based randomisation approach protected node identifications and sensitive links and compared this approach with $k$-degree anonymity approaches. For improving data utility, many constrained modification approaches were designed. Liu and Terzi (2008) constructed a $k$-degree anonymous network through integer linear programming and edge switching. Casas-Roma et al. (2017) obtained a $k$-degree anonymous network with the minimum number of edge
(NE) modified. Macwan and Patel (2018) proposed a $k$-anonymity approach to keep at least $k$-elements of mutual friends holding the same value to get better data utility.

Compared with edge and vertex modification approaches, generalisation approaches can achieve more stricter privacy preserving. Hay et al. (2008) applied structural generalisation approaches to obtain a vertex $k$-anonymous super-graph by clustering nodes and edges. In $\mathrm{Yu}$ et al. (2018), a clustering perturbation algorithm based on local clustering and modifying edges was proposed to decrease the privacy disclosure of vertices and community structures.

Different from these two kinds of graph modification approaches, the uncertain graph approach takes advantage of the uncertainty to modify an original graph, which can better keep the structure of original graph. In the uncertain graph approaches, Boldi et al. (2012) introduced the concept of uncertain graph for privacy preserving and proposed $(k, \varepsilon l)$-obfuscation approach, where $k$ is the desired obfuscation level and $\varepsilon l$ is a tolerance parameter, which assigned an specific probability to each edge in the network graph. A Rand-Walk algorithm based on random walks to construct an uncertain graph was introduced by Mittal et al. (2013), which have outstanding privacy preserving. For achieving better utility, Nguyen et al. (2014) presented a maximum variance approach that keep the degree of original graph unchanged when generating an uncertain graph. Then, Nguyen et al. (2015) also proposed a new approach that takes advantage of the uncertain adjacency matrices to get a generalised obfuscation model. Furthermore, for better understanding the uncertain graph, we present one example illustrated in Figure 1.

However, owing to the insufficient privacy preserving, those approaches can not effectively withdraw attacks based on background knowledge. To solve this problem, the differential privacy developed by Dwork (2006) has been extensively used for preserving the graph data, which was usually classified into two categories: edge-DP and node-DP. Hay et al. (2009) proposed an efficient algorithm to release the degree distribution of a network. By using edge the differential privacy, Hay et al. (2010) applied the constrained inference technique to release a private estimate of degree distribution in graph. In Qian et al. (2018), the goal was to publish a histogram of node strength which was close to the true distribution of graph, while satisfying the 
edge differential privacy. In weighted social graphs, Li et al. (2017) use the merging barrels and consistency inference strategy to improve the data utility of the perturbed graph. To enhance privacy preserving, Day et al. (2016) proposed two approach under node-DP by applying the aggregation and cumulative histogram to release the degree distribution. For publishing an approximation to a linear statistic, Chen and Zhou (2013) presented a novel mechanism based on node-DP. In Kasiviswanathan et al. (2013), a node differential privacy algorithm was designed by using several techniques which mapped the original graph into the set of graphs.

Figure 1 An example of constructing an uncertain graph

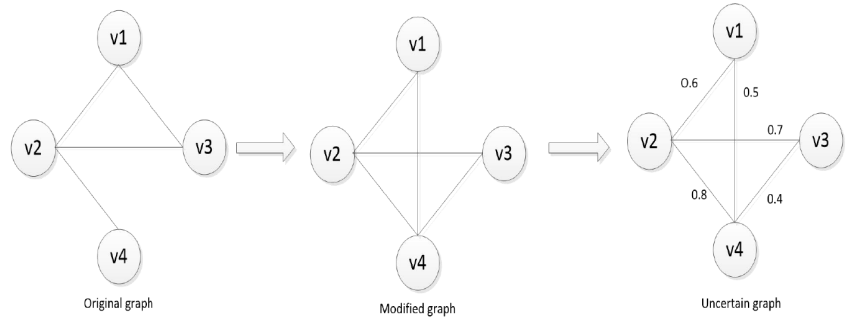

More importantly, as an input perturbation algorithm which perturbed the input value by a probability mechanism, the randomised response was widely adapted and studied. In Van den Hout and Van der Heijden (2002), a randomised response method was used to control statistical disclosures when a dataset was released. The randomised response had also been used to release networks data under $\varepsilon$-edge differential privacy in Karwa et al. (2014), which offers rigorous privacy guarantees for an original network.

As a special kind of data, the graph data in social networks has a particular characteristic of link relationship between two members, which draws more and more attention in data mining. To achieve privacy preserving for this particular characteristic, we combine random response with differential privacy to construct an uncertain graph. Therefore, we extend the research in uncertain graphs and introduce a new approach including two parts: edge modification and injection uncertainty, which satisfies differential privacy while providing better data utility. In this approach, a modification of edges based on random response (MERR) algorithm is proposed, which modifies edges of original graph according to a randomised response mechanism, while a injection of uncertainty based on $k$-edges differential privacy (IUDP) algorithm is designed to provide rigorous privacy guarantees for edges. Furthermore, duo to the uncertainty of edges in uncertain graph, the expectation of editing distance of graph is devised to measure privacy preserving.

\section{Preliminaries}

In this paper, we abstract a social network as a simple undirected graph $G=(V, E)$, where $V$ denotes nodes and $E$ represent edges.

\section{Definition 1 [uncertain graph (Boldi et al., 2012)]}

Let a graph $G=(V, E)$, a function $P: E_{P} \rightarrow[0,1]$, which assigns probabilities to edges in $E$, we can get an uncertain graph $G^{\prime}=\left(V, E^{\prime}, E_{P}\right)$, where $E^{\prime}$ is attained by modifying the $E$ and $E_{P}$ represents the probabilities of edges.

Compared with a graph $G$, the uncertain graph $G^{\prime}$ has the same nodes as $G$ and has different edges from $G$. In a deterministic graph, the probabilities of all edges are 1 .

\section{Definition 2 [neighbouring graph (Hay et al., 2009)]}

Given two graphs $G_{a}=\left(V_{a}, E_{a}\right)$ and $G_{b}=\left(V_{b}, E_{b}\right), G_{a}$ and $G_{b}$ are neighbours if $\left|V_{a} \oplus V_{b}\right|+\left|E_{a} \oplus E_{b}\right|=1$, where $\oplus$ is exclusive - OR operation.

Assuming $V_{a}=V_{b}$, we can describe the Definition 2 as that $G_{a}$ and $G_{b}$ are neighbours if $\left|E_{a} \oplus E_{b}\right|=1$, the Hamming distance between $G_{a}$ and $G_{b}$ is 1 . As shown in Figure 2 . Figure 2(a) is the original graph, according to the Definition 2, Figure 2(a) and Figure 2(b) are neighbouring graphs.

Figure 2 An example of the neighbouring graphs

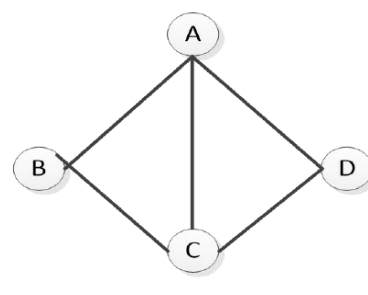

(a)

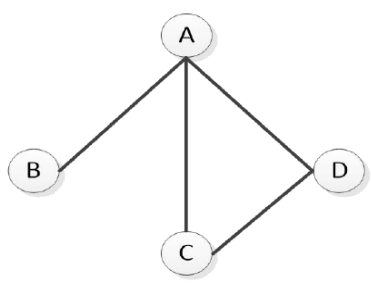

(b)

\section{Definition 3 [sensitivity (Dwork, 2006)]}

Let $F$ be a sequence of queries: $G \rightarrow G$, the sensitivity of $F$ is:

$$
\Delta f=\operatorname{Max}_{G_{a}, G_{b}}\left\|F\left(G_{a}\right)-F\left(G_{b}\right)\right\|_{1}
$$

The Hamming distance is used to calculate the sensitivity of $F$. Because $G_{a}$ is different from $G_{b}$ by one edge, we can get the sensitivity of $F$ is equal to 1 .

\section{Definition 4 [differential privacy (Dwork, 2006)]}

If a algorithm $Z$ is $\varepsilon$-differential privacy for all outputs $S \subseteq$ Range( $Z$ ), we can get the result as follow:

$$
P_{r}\left[Z\left(G_{a}\right) \in S\right] \leq \exp (\varepsilon) \times P_{r}\left[Z\left(G_{b}\right) \in S\right]
$$

where $G_{a}$ and $G_{b}$ are neighbours and $\varepsilon$ is a privacy preserving level. 
Figure 3 The model of constructing an uncertain graph

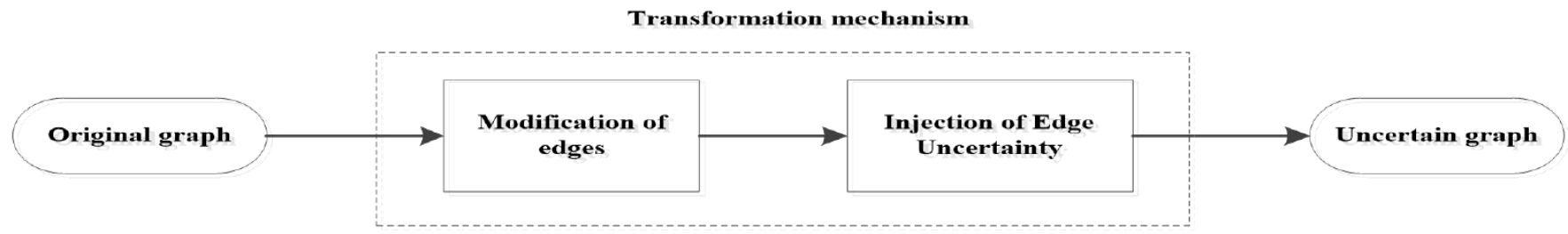

\section{Definition 5 [Laplace mechanism (Dwork, 2006)]}

For a sequence of queries $F: G \rightarrow G$, an algorithm $Z$ will satisfy $\varepsilon$-differential privacy if the following holds:

$$
Z(G)=F(G)+\operatorname{Lap}(\Delta f / \varepsilon)
$$

where $\mu=0, m=\Delta f / \varepsilon$ and $\operatorname{Lap}(\Delta f / \varepsilon)$ represents the Laplace noise.

In the Laplace mechanism, the Laplace noise distribution is shown in equation (1).

$$
n(x)=\frac{1}{2 s} \exp \left(-\frac{|x-\mu|}{s}\right)
$$

where $\mu$ is a position parameter, $s$ denotes a scale parameter and $x$ is a random variable.

The cumulative distribution of Laplace noise distribution can be seen in equation (2).

$$
H_{n}\left(\beta_{i}\right)=\int_{-\infty}^{\beta_{i}} n(x) d x
$$

\section{Definition 6 [k-edge-differential privacy (Hay et al., 2009)]}

Given two graphs $G_{a}=\left(V_{a}, E_{a}\right)$ and $G_{b}=\left(V_{b}, E_{b}\right), G_{a}$ and $G_{b}$ are neighbours if $\left|E_{a} \oplus E_{b}\right|=k$, where $\oplus$ is exclusive OR operation, $1<k<|E|$.

When $1<k<|E|, k$-edges-differential privacy can achieve stronger privacy preserving than edge-differential because its sensitivity is $k$.

\section{Definition 7 [randomised response (Holohan et al., 2017)]}

$X_{i}: \Omega \rightarrow\{0,1\}$ is a random variable for each $i \in N$, dependents on the real value $x_{i}$. The randomised response mechanism is defined as follow:

$$
P\left(X_{i}=k \mid x_{i}=j\right)=p_{j k}
$$

A randomised response mechanism is uniquely determined by its design matrix $\boldsymbol{P}_{r r}, p_{00}$ indicates the probability that the random output equals 0 when the real value is $0, p_{01}$ denotes the probability that the random output equals 1 when the real value is 0 .

$$
P_{r r}=\left(\begin{array}{cc}
P_{00} & P_{01} \\
P_{10} & P_{11}
\end{array}\right) \quad P_{00}, P_{11} \in[0,1]
$$

Definition 8 [randomised response satisfying $\varepsilon$-differential privacy (Holohan et al., 2017)]

Given a parameter $\varepsilon$, the randomised response scheme following the design matrix $P$ in Definition 7 will achieve $\varepsilon$-differential privacy if:

$$
\max \left(\frac{p_{00}}{p_{01}}, \frac{p_{11}}{p_{10}}\right) \leq e^{\varepsilon}
$$

\section{Definition 9 [post-processing (Dwork, 2006)]}

Given a randomised algorithm $M$ that satisfies $\varepsilon$-differential privacy, we can preserve the graph $G$ by using the algorithm $M$ and get the output of the algorithm $M$, which denotes $G^{\prime}$. Let $N$ be an arbitrary randomised mapping, when $N$ is applied on $G^{\prime}$ to attain $G^{\prime \prime}$, the algorithm $M \circ N: G \rightarrow G^{\prime \prime}$ satisfies $\varepsilon$-differential privacy.

\section{Definition 10 (sequence combination)}

Given a dataset $D$ and $n$ privacy algorithms $\left\{M_{1}, M_{2}, \ldots\right.$, $\left.M_{n}\right\}, M_{i} \quad\{1 \leq i \leq n\}$ satisfies $\varepsilon$-differential privacy, the sequence combination $\left\{M_{1}, M_{2}, \ldots, M_{n}\right\}$ satisfies $\sum_{i}^{n} \varepsilon_{i}$ differential privacy.

\section{Model and algorithm}

\subsection{Model of the approach}

As shown in Figure 3, a model is devised to construct an uncertain graph. The key of this model is transformation mechanism which is consists of two parts: modification of edges and injection of uncertainty. Under this model, MERR algorithm can modify the graph according to random response mechanism and IUDP algorithm injects the uncertainty to edges of a modified graph.

\subsection{MERR algorithm}

In order to better understand the MERR algorithm we proposed, we give the model of the algorithm which can be seen in Figure 4.

In Figure 4, it can be see that the model of MERR algorithm has three parts. Firstly, we generate a set of edges from original graph as the input of random response mechanism. Secondly we obtain a modified graph by random response mechanism. Finally, in order to improve 
the data utility, we use post-processing to add edges to the modified graph.

\section{Algorithm 1 MERR algorithm}

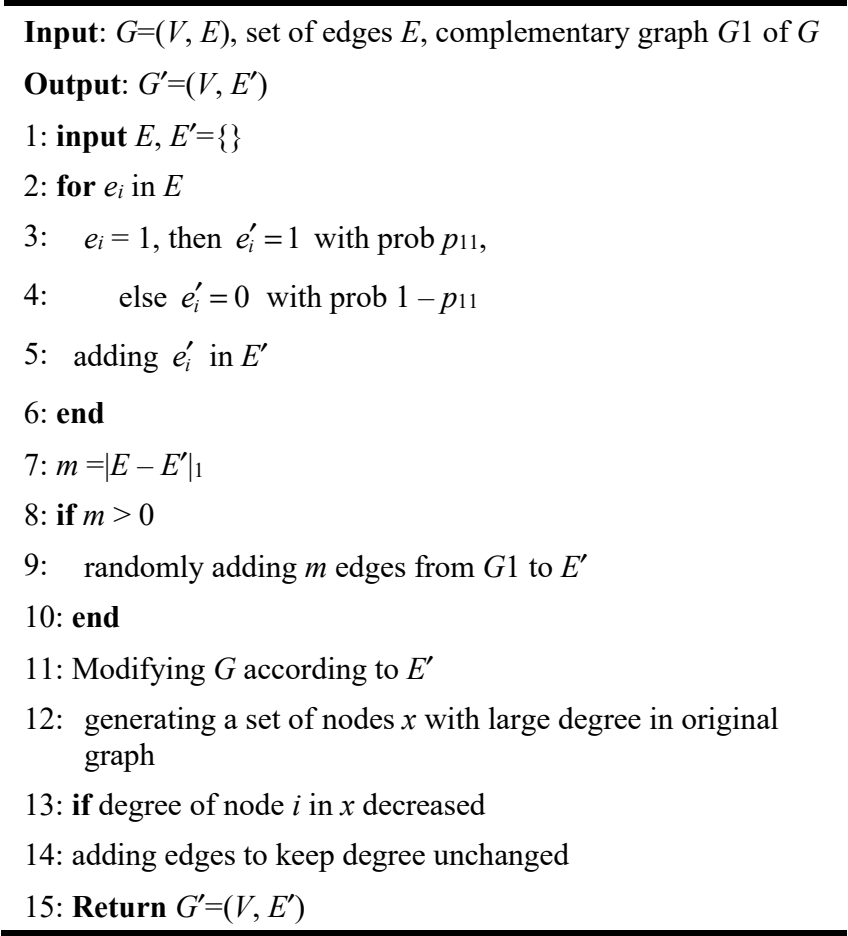

The MERR algorithm can be seen in Algorithm 1. The input of MERR algorithm is a set of edges $E$ of an original graph and an empty set $E^{\prime}$ which are outlined in line 1 . Line 3 to line 5 generates a set of $E^{\prime}$ by using random response mechanism, which is different from the set $E$. Line 7 calculates the distance between set $E$ and set $E^{\prime}$ according to $\left|E-E^{\prime}\right|_{1}$. Lines 8-10 randomly adds $m$ edges in $E^{\prime}$ to keep the $N E \mathrm{~s}$ in the original graph unchanged. For preserving the degree of some nodes unchanged, we adds some edges to improve data utility in line 11-line 14 .

Finally, MERR algorithm returns a modified graph with better utility in line 15 .

\subsection{IUDP algorithm}

For the sake of constructing an uncertain graph, we devise IUDP algorithm which is showed in Figure 5.

In Figure 5, we describe the IUDP algorithm in three steps:

1 we get the noise $n=\left(n_{1}, n_{2}, \ldots, n_{i}, \ldots, n_{N}\right)$ generated by $k$-edges differential privacy, where $N$ is the $N E \mathrm{~s}$, then generate a noise graph by assigning each edge a noise value $n_{i}$

2 according to the principle of post processing, we calculate the probability $p_{i}$ of a noise $n_{i}$, where $p_{e i}=P_{n}\left[n_{i}\right]=H_{n}\left(n_{i}\right), p_{e i} \in P(0,1)$

3 we assign the probability $p_{e i}$ to each edge of $G^{\prime}$.

In the end, we get an uncertain graph $G^{\prime \prime}$ which preserves the original graph.
Algorithm 2 IUDP algorithm

$$
\begin{aligned}
& \text { Output: } G^{\prime \prime}=\left(V, E^{\prime \prime}, E_{P}\right) \\
& \text { 1: } s \leftarrow \Delta f / \varepsilon \\
& \text { 2: for } e_{i} \text { in } E^{\prime}: \\
& \text { 3: } \quad n_{i} \leftarrow L a p(s) \\
& \text { 4: } \quad \text { If } n_{i}<0 \\
& \text { 5: } \quad n_{i}=\left|n_{i}\right| \\
& \text { 6: end } \\
& \text { 7: } \quad P_{n}\left[n_{i}\right] \leftarrow H_{n}\left(n_{i}\right) \\
& \text { 8: } \quad p_{e i} \leftarrow P_{n}\left[n_{i}\right] \\
& \text { 9: } \quad e_{i} \leftarrow p_{e i} \\
& \text { 10: } \quad \text { adding } p_{e i} \text { in } E_{P} \\
& \text { 11: end }
\end{aligned}
$$$$
\text { Input: } G^{\prime}=\left(V, E^{\prime}\right) \text {, sensitivity } \Delta f=k \text { and privacy budget } \varepsilon
$$

The IUDP algorithm is described in Algorithm 2. When we input a modified graph $G^{\prime}$, sensitivity $\Delta f$ and privacy budget $\varepsilon$, the output of IUDP algorithm is an uncertain graph. In line 1 , the scale parameter $\mathrm{s}$ is $\Delta f / \varepsilon$, where $\Delta f$ is equal to $k$. Line 3 to line 6 generates the noise value $n$ by Laplace distribution. If $n_{i}$ is less than 0 , we take its absolute value. Line 7 calculates the probability value corresponding to the noise value according to equation (2), lines 8-10 assigns the probability value to each edge of the graph and gets a set $P$ which contains the probability value of edges. Finally, IUDP algorithm output an uncertain graph in line 12, which preserves the privacy of the original graph.

\subsection{Analysis of approach}

\subsubsection{Analysis of MERR algorithm}

When a randomised response mechanism satisfies $\varepsilon$-differential privacy, we get a design matrix as follow:

$$
P_{r r}=\left(\begin{array}{c}
\frac{e^{\varepsilon}}{1+e^{\varepsilon}} \frac{1}{1+e^{\varepsilon}} \\
\frac{1}{1+e^{\varepsilon}} \frac{e^{\varepsilon}}{1+e^{\varepsilon}}
\end{array}\right)
$$

In this design matrix, we will get $p_{11}=\frac{e^{\varepsilon}}{1+e^{\varepsilon}}, p_{10}=\frac{1}{1+e^{\varepsilon}}$.

Especially, we can get a sequence $Q\left(s_{1}, s_{2}, \ldots, s_{n}\right)$ according to a set of edges $E, s_{i}=1, i=1, \ldots, n$, since there is an edge existing in graph.

Theorem 1: The MERR algorithm satisfies $\varepsilon$-differential privacy.

Proof: Let $R$ denotes a randomised response mechanism, $P_{r}[x \rightarrow y]$ represents the probability that $x \in\{0,1\}$ changes to $y \in\{0,1\}, q=1-p=\frac{e^{\varepsilon}}{1+e^{\varepsilon}}$. When $\varepsilon>0$, we get $q>p$. 
Figure 4 The MERR algorithm

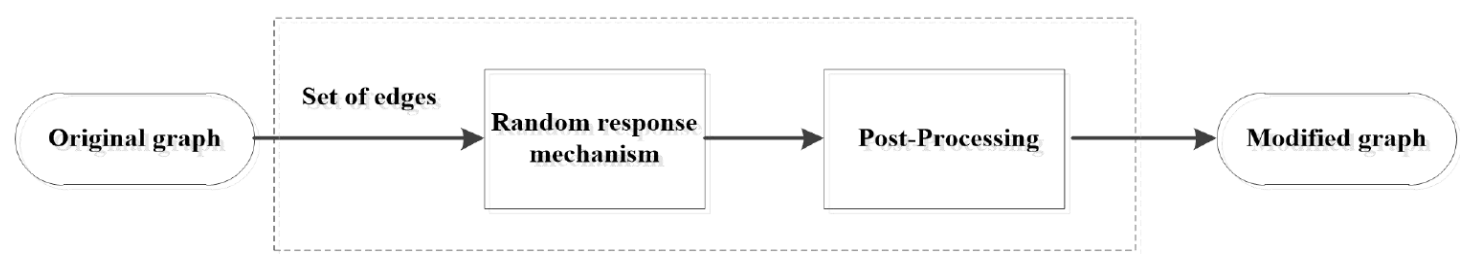

Figure 5 The IUDP algorithm

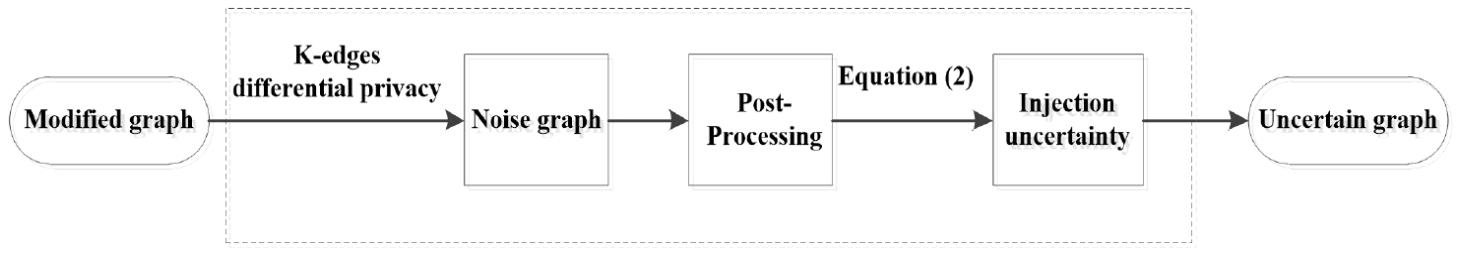

Let $Q\left(s_{1}, s_{2}, \ldots, s_{n}\right)$ and $Q^{\prime}\left(s_{1}^{\prime}, s_{2}^{\prime}, \ldots, s_{n}^{\prime}\right)$ be neighbour sequences that there is one different element between them. Without loss of generality, assume $S_{1} \neq S_{1}^{\prime}$, let $M\left(m_{1}, m_{2}, \ldots, m_{n}\right)$ be any output of $R$, we will get:

$$
\begin{aligned}
& \frac{p_{r}[R(Q)=M]}{p_{r}\left[R\left(Q^{\prime}\right)=M\right]} \\
= & \frac{p_{r}\left[s_{1} \rightarrow m_{1}\right] \cdots p_{r}\left[s_{n} \rightarrow m_{n}\right]}{p_{r}\left[s_{1}^{\prime} \rightarrow m_{1}\right] \cdots p_{r}\left[s_{n}^{\prime} \rightarrow m_{n}\right]} \\
= & \frac{p_{r}\left[s_{1} \rightarrow m_{1}\right]}{p_{r}\left[s_{1}^{\prime} \rightarrow m_{1}\right]} \leq \frac{q}{p}=e^{\varepsilon}
\end{aligned}
$$

In accordance with the principle of post-processing, it is evident that the MERR algorithm satisfies $\varepsilon$-differential privacy. In addition, for improving the data utility, we limit the $N E$ s added and keep the degree of some nodes unchanged.

\subsubsection{Analysis of IUDP algorithm}

Theorem 2: The IUDP algorithm satisfies $\varepsilon$-differential privacy.

Proof: Given two graphs $G_{a}, G_{b}$ which are neighbours, the Hamming distance between $G_{a}$ and $G_{b}$ is $k$. Let $F(\cdot)$ be some identity mapping $F: G \rightarrow G$. so $F\left(G_{a}\right) \rightarrow G_{a}, F\left(G_{b}\right) \rightarrow G_{b}$. According to Definition 3, we will get the sensitivity of $F$ :

Then according to the Laplace mechanism, the Laplace noise is add to the output of $F$, where $L M$ represents the Laplace mechanism, $\eta$ denotes the Laplace noise and $Z$ is the result we get:

$$
\begin{aligned}
& Z=L M\left(G_{a}\right)=F\left(G_{a}\right)+\eta \\
& Z=L M\left(G_{b}\right)=F\left(G_{b}\right)+\eta \\
& Z=\left(z_{1}, z_{2}, \ldots, z_{n}\right) \\
& \Delta f=\max _{G 1, G 2}|| F\left(G_{a}\right)-F\left(G_{b}\right) \|_{1} \\
& \Delta f=\left|f_{1}\left(G_{a}\right)-f_{1}\left(G_{b}\right)\right|+\left|f_{2}\left(G_{a}\right)-f_{2}\left(G_{b}\right)\right| \\
& +\ldots+\left|f_{n}\left(G_{a}\right)-f_{n}\left(G_{b}\right)\right| \\
& =k
\end{aligned}
$$

Let $P_{r}[Z(G a)]$ represents the probability density function of $\operatorname{LM}(G 1, F, \varepsilon)$, and $\operatorname{P}_{r}[Z(G b)]$ denotes the probability density function of $\operatorname{LM}(G 2, F, \varepsilon)$. The proof is showed as follows:

$$
\begin{aligned}
& \frac{P_{r}\left[Z\left(G_{a}\right)\right]}{P_{r}\left[Z\left(G_{b}\right)\right]}=\frac{P_{r}\left[L M\left(G_{a}\right)\right]}{P_{r}\left[L M\left(G_{b}\right)\right]}=\frac{P_{r}\left[\eta\left(G_{a}\right)\right]}{P_{r}\left[\eta\left(G_{b}\right)\right]} \\
& =\frac{P_{r}\left[Z-F\left(G_{a}\right)\right]}{P_{r}\left[Z-F\left(G_{b}\right)\right]}=\frac{\frac{1}{\frac{\Delta f}{\varepsilon}} \exp \left(-\frac{\left|Z-F\left(G_{a}\right)\right|}{\frac{\Delta f}{\varepsilon}}\right)}{\frac{1}{2 \frac{\Delta f}{\varepsilon}} \exp \left(-\frac{\left|Z-F\left(G_{b}\right)\right|}{\frac{\Delta f}{\varepsilon}}\right)} \\
& =\frac{\exp \left(-\frac{\varepsilon\left|Z-F\left(G_{a}\right)\right|}{\Delta f}\right)}{\exp \left(-\frac{\varepsilon\left|Z-F\left(G_{b}\right)\right|}{\Delta f}\right)} \\
& =\frac{\exp \left(-\frac{\varepsilon\left|z_{1}-f_{1}\left(G_{a}\right)\right|}{\Delta f}\right) \cdots \exp \left(-\frac{\varepsilon\left|z_{n}-f_{n}\left(G_{a}\right)\right|}{\Delta f}\right)}{\exp \left(-\frac{\varepsilon\left|z_{1}-f_{1}\left(G_{b}\right)\right|}{\Delta f}\right) \cdots \exp \left(-\frac{\varepsilon\left|z_{n}-f_{n}\left(G_{b}\right)\right|}{\Delta f}\right)} \\
& =\prod_{i=1}^{n} \exp \left(\frac{\varepsilon\left|z_{i}-f_{i}\left(G_{a}\right)\right|}{\Delta f}-\frac{\varepsilon\left|z_{i}-f_{i}\left(G_{b}\right)\right|}{\Delta f}\right) \\
& =\prod_{i=1}^{n} \exp \left(\frac{\varepsilon\left(\left|z_{i}-f_{i}\left(G_{a}\right)\right|-\left|z_{i}-f_{i}\left(G_{b}\right)\right|\right)}{\Delta f}\right) \\
& \leq \prod_{i=1}^{n} \exp \left(\frac{\varepsilon\left|f_{i}\left(G_{a}\right)-f_{i}\left(G_{b}\right)\right|}{\Delta f}\right) \\
& =\exp \left(\frac{\varepsilon\left(\left|f_{1}\left(G_{a}\right)-f_{1}\left(G_{b}\right)\right|+\ldots+\left|f_{n}\left(G_{a}\right)-f_{n}\left(G_{b}\right)\right|\right)}{\Delta f}\right) \\
& \leq \exp \left(\frac{\varepsilon \cdot \Delta f}{\Delta f}\right)=e^{\varepsilon}
\end{aligned}
$$

After getting a noise graph, we convert the noise graph into an uncertain graph by assigning the probability to each edge of the graph. In accordance with the principle 
of post-processing, we can know that the process of transforming a noise graph into an uncertain graph satisfies $\varepsilon$-differential privacy. Therefore, we can make a conclusion that our IUDP algorithm satisfies $\varepsilon$-differential privacy. Furthermore, with more edges added in MERR algorithm and a bigger probability assigned to each edge, IUDP algorithm will gain better data utility.

In summary, owing to the fact that MERR algorithm and IUDP algorithm satisfy $\varepsilon$-differential privacy, according to the principle of sequence combination in differential privacy, our approach based on these two algorithms satisfies $\varepsilon$-differential privacy.

\section{Experiments}

\subsection{Dataset}

In experiments, two kinds of experiment data are utilised, which include the synthetic datasets and the real datasets. The synthetic datasets are obtained from ER graphs, which contain 200 and 500 nodes. The real datasets contains dolphin clubs with 62 nodes, Hamsterster friendships with 1,858 nodes and Facebook with 4,039 nodes.

For the purpose of evaluating our approach, $(k, \varepsilon l)$-obfuscation approach and Rand-Walk approach are utilised for the comparison. All simulation experiments run on a HP computer, which has Intel Core i5-8500 with $3.00 \mathrm{GHz}$ and $12 \mathrm{~GB}$ memory. For programming, Python and MATLAB are used on the Microsoft Windows 7 operating system.

\subsection{Privacy evaluation}

\subsubsection{Privacy measurement}

In our approach, when we get an uncertain graph $G_{u}$, the expectation of editing distance between $G_{u}$ and the deterministic graph $G$ is:

$$
\begin{aligned}
& \operatorname{EED}\left(G, G_{u}\right)=\operatorname{EED}\left(G, G^{\prime}\right)+\operatorname{EED}\left(G^{\prime}, G_{u}\right) \\
& =m_{k}+\sum_{e_{i} \in G^{\prime}}\left(1-p_{i}\right)+\sum_{e_{i} \notin G^{\prime}} p_{i}
\end{aligned}
$$

where $G^{\prime}$ is obtained by our MERR algorithm, $e_{i}$ belongs to the edges set of $G_{u}, p_{i}$ is the probability of the edge $e_{i}, m_{k}$ equals the edit distance between two deterministic graphs $G$ and $G^{\prime}$, which is calculated with the following formula:

$$
m_{k}=G_{E_{k}}+x_{k}
$$

where $G_{E_{k}}$ denotes the number edges which is deleted in a random response in a randomised response, $x_{k}$ represents the number of modified edges in the selected nodes.

The expectation of editing distance $(E E D)$ is showed as follows:

$$
E E D\left(G, G_{u}\right)=m_{k}+\sum_{e_{i} \in G^{\prime}} E E D_{1}+\sum_{e_{i} \notin G^{\prime}} E E D_{2}
$$

\subsubsection{Privacy analysis}

In order to evaluate the different uncertain graphs approaches, we use the $E E D$ to measure the privacy preserving. The greater $E E D$, the better privacy preserving an uncertain graph approach achieves. We execute all datasets ten times by using our approach and other approaches to average out the results.

In the comparative experiments, the parameter of three approaches is showed in Table 1 . In $(k, \varepsilon l)$-obfuscation approach, the obfuscation level $k$ belongs to $\{10,20\}$, tolerance parameter $\varepsilon l$ equals 0.1 , multiplier factor $c$ is 1 and white noise $q$ is equal to 0.01 . In Rand-Walk approach, the parameter $t$ denotes the size of noise. In our approach, the parameter $\varepsilon$ is the privacy budget which in $\{0.1,1,2\}$.

The result of EED values can be seen in Table 1 . In Table 1, the EED values of our approach is showed from the first to the third row, where the $E E D$ values increase as the value of $\varepsilon$ decreases, which means that the privacy preserving of our approach becomes stronger concurrently. When the number of nodes increase, we can see that the $E E D$ in our approach raise simultaneously, which indicates that the uncertain graph obtained by this approach is more and more different from the original graphs, that is, the better privacy preserving for the original graph.

As show in Table 1, the EED values of $(k, \varepsilon l)$-obfuscation approach is in fourth line to the fifth row while the sixth line to the seventh row indicate the $E E D$ values of Rand-Walk approach. By comparing the $E E D$ values of different approaches in same datasets, we can see in Table 1 that the EED value of our approach is larger than $(k, \varepsilon l)$-obfuscation approach while is smaller than Rand-Walk approach. In addition, the EED values of Rand-Walk approach is the largest.

In the light of the result of comparison, there is a conclusion that our approach can provides better privacy preserving than $(k, \varepsilon l)$-obfuscation approach, but is weaker than Rand-Walk approach.

\subsection{Utility evaluation}

\subsubsection{Utility metrics}

In the beginning, in order to evaluate the data utility, the $N E$, average of degree $(A D)$ and degree variance $(D V)$ is used in our experiments.

Then, we also make use of several structural measures, such as the diameter $\left(S_{\text {Diam }}\right)$ and the average distance $\left(S_{A P D}\right)$, which represents the character of distance. 
Table 1 The EED values in different approaches

\begin{tabular}{lcccccc}
\hline Node number & $n$ & $n=500$ & $n=1,000$ & $n=62$ & $n=1,858$ & $n=4,309$ \\
\hline Our approach & $\varepsilon=0.1$ & $31,131.68$ & $125,041.78$ & 199.55 & $53,227.33$ & $237,742.05$ \\
& $\varepsilon=1$ & $31,056.84$ & $124,720.70$ & 197.86 & $53,510.07$ & $237,789.76$ \\
& $\varepsilon=2$ & $31,033.35$ & $124,284.15$ & 194.97 & $53,499.91$ & $233,354.40$ \\
\multirow{3}{*}{$(k, \varepsilon l)$-obfuscation } & $k=10$ & 35.35 & 221.36 & 698.99 & $1,395.07$ & $6,252.51$ \\
& $k=20$ & 33.42 & 225.57 & 697.56 & $1,413.62$ & $6,202.99$ \\
Rand-Walk & $t=5$ & $1,959.27$ & $12,303.99$ & $42,459.23$ & $79,936.99$ & $385,147.79$ \\
& $t=10$ & $1,932.32$ & $12,285.31$ & $42,347.47$ & $79,670.20$ & $385,047.41$ \\
\hline
\end{tabular}

Last but not least, we can utilise utility to measure data utility, which described as following:

$$
\text { Utility }=U V / R V
$$

where $U V$ is the graph metrics in uncertain graphs achieved by the privacy preserving approaches and $R V$ is the real utility metrics in original graphs.

The greater the value of utility, the better the data utility.

In addition, for comparing the data utility of different approaches, we compute the error on these graph metrics as follows:

$$
\Delta q\left(G, G_{u}\right)=\left|m(G)-m\left(G_{u}\right)\right|
$$

where $m$ is one of the graph utility metrics, $G$ is the original graph and $G_{u}$ is an uncertain graph. The less $\Delta q$, the better data utility.

Figure 6 The utility of $N E$ and $A D$ in our algorithm, (a) the utility of $N E$ (b) the utility of $A D$ (see online version for colours)

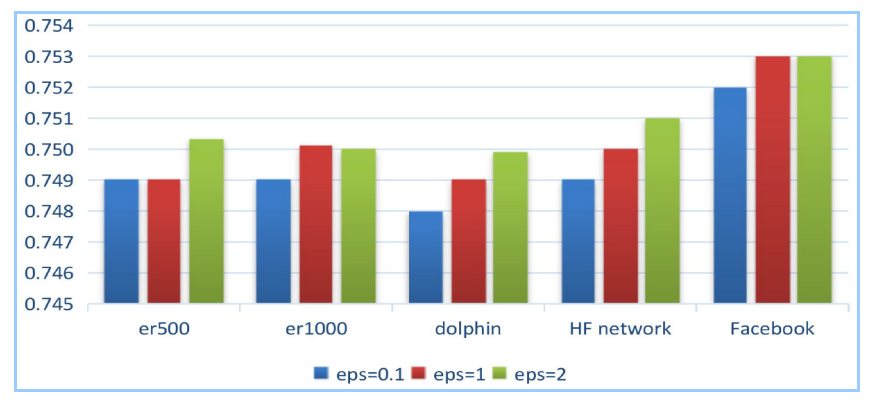

(a)

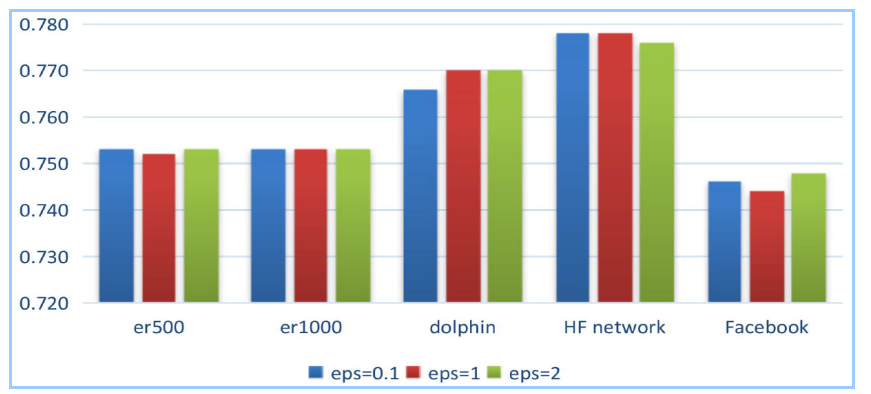

(b)

)

\subsubsection{Utility analysis}

In utility analysis, Table 2 with $\Delta f=8$ shows the utility metrics in original graph and our approach. Table 3 gives the utility metrics in original graph, $(k, \varepsilon l)$-obfuscation approach and Rand-Walk approach.

\begin{tabular}{|c|c|c|c|c|c|}
\hline \multirow{2}{*}{$\begin{array}{l}\text { Nodes' } \\
\text { number }\end{array}$} & \multirow{2}{*}{ Metrics } & \multirow{2}{*}{$\begin{array}{c}\text { Original } \\
\text { graph }\end{array}$} & \multicolumn{3}{|c|}{$\varepsilon=(\varepsilon 1+\varepsilon 2) \Delta f=8$} \\
\hline & & & $\varepsilon=0.1$ & $\varepsilon=1$ & $\varepsilon=2$ \\
\hline \multirow[t]{5}{*}{$n=500$} & $N E$ & 24,844 & $18,631.79$ & $18,628.57$ & $18,636.61$ \\
\hline & $A D$ & 99 & 74.52 & 74.51 & 74.54 \\
\hline & $D V$ & 73.26 & $2,020.41$ & $2,016.23$ & $2,003.86$ \\
\hline & $S_{\text {Diam }}$ & 4 & 4 & 4 & 4 \\
\hline & $S_{A P D}$ & 1.80 & 1.84 & 1.84 & 1.84 \\
\hline \multirow[t]{5}{*}{$n=1,000$} & $N E$ & 99,902 & $74,925.09$ & $74,948.40$ & $74,946.77$ \\
\hline & $A D$ & 199 & 149.85 & 149.89 & 149.89 \\
\hline & $D V$ & 151.45 & $7,936.28$ & $7,990.28$ & $7,904.30$ \\
\hline & $S_{\text {Diam }}$ & 4 & 4 & 4 & 4 \\
\hline & $S_{A P D}$ & 1.80 & 1.87 & 1.88 & 1.86 \\
\hline \multirow[t]{5}{*}{$n=62$} & $N E$ & 159 & 119.02 & 119.38 & 119.83 \\
\hline & $A D$ & 5 & 3.83 & 3.85 & 3.86 \\
\hline & $D V$ & 8.61 & 20.13 & 21.67 & 18.53 \\
\hline & $S_{\text {Diam }}$ & 10 & 10 & 10 & 10 \\
\hline & $S_{A P D}$ & 3.35 & 3.53 & 3.56 & 3.36 \\
\hline \multirow[t]{5}{*}{$n=1,858$} & $N E$ & 12,534 & $9,396.62$ & $9,398.62$ & $9,410.62$ \\
\hline & $A D$ & 13 & 10.12 & 10.12 & 10.10 \\
\hline & $D V$ & 430.02 & $1,737.64$ & $1,725.65$ & $1,710.78$ \\
\hline & $S_{\text {Diam }}$ & 4 & 4 & 4 & 4 \\
\hline & $S_{A P D}$ & 3.39 & 2.92 & 2.92 & 2.92 \\
\hline \multirow[t]{5}{*}{$n=4,039$} & $N E$ & 88,234 & $66,321.56$ & $66,452.69$ & $66,504.76$ \\
\hline & $A D$ & 44 & 32.84 & 32.75 & 32.93 \\
\hline & $D V$ & $3,262.12$ & $2,789.76$ & $2,773.89$ & $2,764.23$ \\
\hline & $S_{\text {Diam }}$ & 4 & 3 & 3 & 3 \\
\hline & $S_{A P D}$ & 3 & 2.81 & 2.81 & 2.81 \\
\hline
\end{tabular}

Table 2 Utility metrics in our approach $(\Delta f=8)$ 
Table 3 Utility metrics in $(k, \varepsilon l)$-obfuscation algorithm and Rand-Walk approach

\begin{tabular}{|c|c|c|c|c|c|c|}
\hline \multirow{2}{*}{$\begin{array}{l}\text { Nodes' } \\
\text { number }\end{array}$} & \multirow{2}{*}{ Metrics } & \multirow{2}{*}{ Original graph } & \multicolumn{2}{|c|}{$k$} & \multicolumn{2}{|c|}{$t$} \\
\hline & & & $k=10$ & $k=20$ & $t=5$ & $t=10$ \\
\hline \multirow[t]{5}{*}{$n=500$} & $N E$ & 24,844 & $20,910.52$ & $20,910.88$ & $12,416.62$ & $12,608.66$ \\
\hline & $A D$ & 99 & 83.64 & 83.65 & 49.66 & 50.44 \\
\hline & $D V$ & 73.26 & $4,393.93$ & $4,391.24$ & 649.02 & 668.30 \\
\hline & $S_{\text {Diam }}$ & 4 & 2 & 2 & 2 & 2 \\
\hline & $S_{A P D}$ & 1.80 & 1.83 & 1.83 & 1.86 & 1.89 \\
\hline \multirow[t]{5}{*}{$n=1,000$} & $N E$ & 99,902 & $76,975.76$ & $77,142.11$ & $50,175.43$ & $50,326.13$ \\
\hline & $A D$ & 199 & 153.95 & 154.28 & 100.35 & 100.65 \\
\hline & $D V$ & 151.45 & $17,794.66$ & $17,750.49$ & $2,585.74$ & $2,602.80$ \\
\hline & $S_{\text {Diam }}$ & 4 & 2 & 2 & 1.8 & 2 \\
\hline & $S_{A P D}$ & 1.80 & 1.82 & 1.82 & 1.89 & 1.89 \\
\hline \multirow[t]{5}{*}{$n=62$} & $N E$ & 159 & 150.60 & 148.76 & 96.89 & 97.77 \\
\hline & $A D$ & 5 & 4.85 & 4.78 & 3.12 & 530.1153 \\
\hline & $D V$ & 8.61 & 15.88 & 16.05 & 5.86 & 5.99 \\
\hline & $S_{\text {Diam }}$ & 10 & 4 & 4 & 4 & 3 \\
\hline & $S_{A P D}$ & 3.35 & 3.36 & 3.42 & 2.79 & 2.57 \\
\hline \multirow[t]{5}{*}{$n=1,858$} & $N E$ & 12,534 & $10,788.40$ & $10,730.92$ & $6,769.34$ & $6,685.62$ \\
\hline & $A D$ & 13 & 11.61 & 12.62 & 7.28 & 7.19 \\
\hline & $D V$ & 430.02 & $1,278.89$ & $1,328.33$ & 122.73 & 119.38 \\
\hline & $S_{\text {Diam }}$ & 4 & 4 & 4 & 3.80 & 3.68 \\
\hline & $S_{A P D}$ & 3.39 & 3.20 & 3.21 & 3.89 & 3.89 \\
\hline \multirow[t]{5}{*}{$n=4,039$} & $N E$ & 88,234 & $86,284.81$ & $86,253.19$ & $45,178.14$ & $45,383.70$ \\
\hline & $A D$ & 44 & 42.72 & 42.71 & 22.37 & 22.47 \\
\hline & $D V$ & $3,262.12$ & $3,245.78$ & $3,246.13$ & 704.29 & 702.23 \\
\hline & $S_{\text {Diam }}$ & 4 & 4 & 4 & 5 & 5 \\
\hline & $S_{A P D}$ & 3 & 3.09 & 3.12 & 3.68 & 3.68 \\
\hline
\end{tabular}

First of all, to illustrate that our approach is available, we use the utility to evaluate the data utility of our approach. In Table 2, most of the utility in our approach can reach $72.0 \%$, the highest is $100 \%$. For instance, we can see the utility over the $S_{\text {Diam }}$ is kept unchanged in Hamsterster friendships dataset.

Furthermore, we use Figure 6 to outlines $N E$ 's and $A D$ 's utility, which indicates our approach can maintain a high utility of the original graphs. As shown in Figure 6(a), the most utility of $N E$ in our approach is more than $74.9 \%$, the highest can reach $75.4 \%$. In Figure 6(b), the highest utility of $A D$ can reach $77.8 \%$, the lowest is $74.8 \%$, while the average utility of $A D$ is more than $74.4 \%$.

Secondly, we compare our approach with $(k, \varepsilon l)$-obfuscation approach and Rand-Walk approach by using the data in Table 2 and Table 3. By analysing data in the tables, we can see that our approach, $(k, \varepsilon l)$-obfuscation approach and Rand-Walk approach have all disturbed the data of original graph, which means these approaches can preserve the original graph. For example, for Facebook dataset, NE of original graph is 88,234 , while $N E$ of three approaches is $66,185.47(\varepsilon=0.1, \Delta f=8), 86,284.81$ $(k=10), 45,178.14(t=5)$ respectively, which a becomes smaller.

In order to demonstrate comparison of three approaches, Figure 7 illustrates the $\Delta q$ in these approaches. Figure 7(a) shows the $\Delta q$ over $A D$ in different approaches, while Figure $7(\mathrm{~b})$ demonstrates the $\triangle q$ over $S_{A P D}$. By comparing $\triangle q$ over $A D$ and $S_{A P D}$, we can see that $(k, \varepsilon l)$-obfuscation approach has better data utility than our approach, while our approach is better than Rand-Walk approach.

At last, by using the experimental results, we can make a conclusion that our approach has a feasible data utility, which can be applied for preserving the link relationship of social networks. 
Figure 7 The comparison of $\Delta q$ in three approaches $(\varepsilon=1, k=20, t=10, \varepsilon l=1)$, (a) the $\Delta q$ of $A D$ (b) the $\triangle q$ of $S A P D$ (see online version for colours)

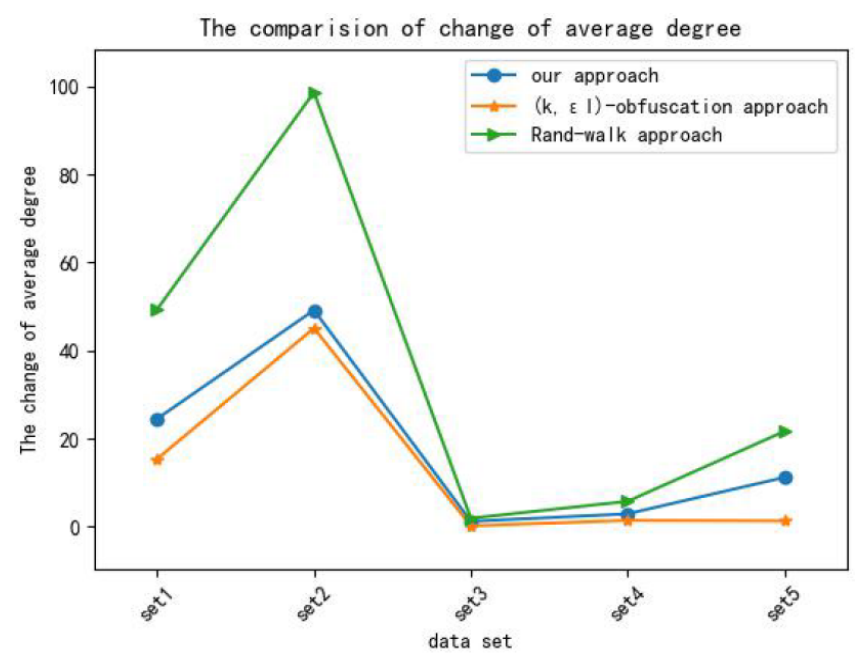

(a)

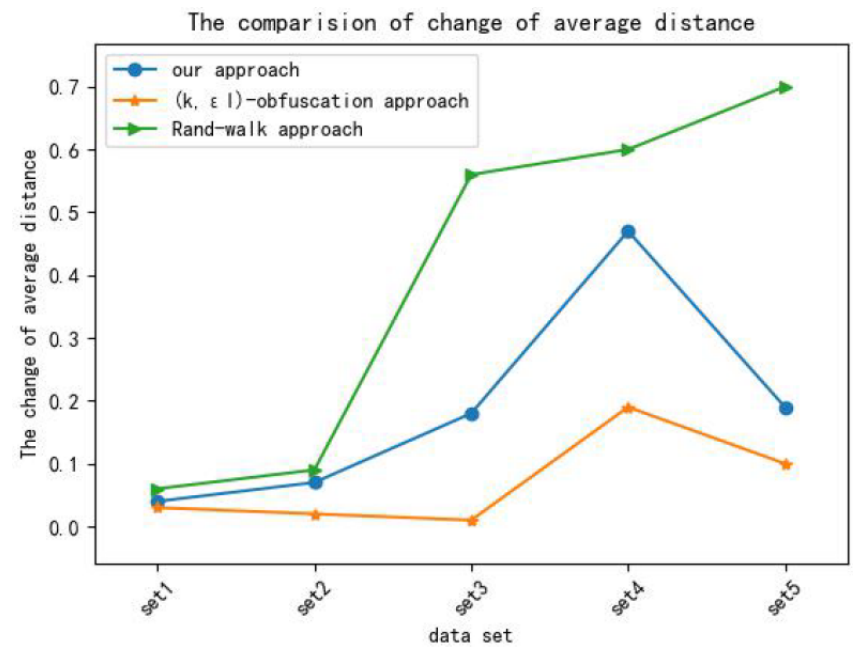

(b)

\section{Conclusions}

In summary, for preserving the link relationship in social networks which is an important structure characteristic of social networks, we take advantage of uncertainty to generate an uncertain graph which can achieve privacy preserving for link relationship while keeping the structure of original social networks. Meanwhile, to resist attacks based on background knowledge, a random response mechanism with differential privacy is adapted to modify edges of graph and the $k$-edges differential privacy is used to inject uncertainty on edges. As a result, an uncertain graph approach based on differential privacy is designed to preserve structure characteristics of social networks, which includes MERR algorithm and IUDP algorithm. In addition, the theoretical analysis proves clearly that our approach satisfies differential privacy, and experiment results indicate that our approach can achieve privacy preserving while attaining better data utility. Consequently, our approach can be widely utilised to preserve privacy of structure data.
In the future, we will focus on the trade off between privacy preserving and data utility to improve our approach.

\section{Acknowledgements}

This research was funded by the National Natural Science Foundation of China (No. 61962033), the Natural Science Basic Research of Shaanxi Province (No. 2020JM-288) and the Fundamental Research Funds for the Central Universities (No. GK201704017, GK201903011).

\section{References}

Backstrom, L., Dwork, C. and Kleinberg, J. (2007) 'Wherefore art thou r3579x?: Anonymized social networks, hidden patterns, and structural steganography', Proc. of 16th Int. Conference on World Wide Web, pp.181-190, DOI: 10.1145/1242572. 1242598.

Boldi, P., Bonchi, F., Gionis, A. et al. (2012) 'Injecting uncertainty in graphs for identity obfuscation', Proc. of the VLDB Endowment, Vol. 5, pp.1376-1387, DOI: 10.14778/2350229. 2350254.

Casas-Roma, J. (2014) 'Privacy-preserving on graphs using randomization and edge-relevance', Proc. of Modeling Decisions for Artificial Intelligence, pp.204-216, DOI: 10.1007/978-3-319-12054-6_18.

Casas-Roma, J., Herrera-Joancomart, J. and Torra, V. (2016) 'A survey of graph-modification techniques for privacy-preserving on networks', Artificial Intelligence Review, Vol. 47, pp.1-26, DOI: 10.1007/s10462-016-9484-8.

Casas-Roma, J., Herrera-Joancomartí, J. and Torra, V. (2017) 'k-degree anonymity and edge selection: improving data utility in large networks', Knowledge and Information Systems, Vol. 50, No. 2, pp.447-474, DOI: 10.1007/s10115016-0947-7.

Chen, S-x. and Zhou, S-g. (2013) 'Recursive mechanism: towards node differential privacy and unrestricted joins', Proc. of ACM SIGMOD Int. Conference on Management of Data, pp.653-664, DOI: 10.1145/2463676.2465304.

Day, W.Y., Li, N. and Lyu, M. (2016) 'Publishing graph degree distribution with node differential privacy', Proc. of 2016 Int. Conference Management of Data, pp.133-138, DOI: $10.1145 / 2882903.2926745$.

Dwork, C. (2006) 'Differential privacy', Proc. of the $33 r$ International Colloquiium on Automata, Languages and Programming, pp.1-12, DOI: 10.1007/11787006_1.

Hay, M., Li, C., Miklau, G. et al. (2009) 'Accurate estimation of the degree distribution of private networks', Proc. of Ninth IEEE Int. Conference on Data Mining Miami Beach, pp.169-178, DOI: 10.1109/ICDM.2009.11.

Hay, M., Miklau, G. and Jensen, D. (2007) 'Anonymizing social networks', $V L D B$, pp.173-187, DOI: 10.1109/SP.2009.22.

Hay, M., Miklau, G., Jensen, D. et al. (2008) 'Resisting structural reidentification anonymized social networks', Proc. of the VLDB Endowment, Vol. 1, pp.102-114, DOI: 10.1007/s00778 $-010-0210-\mathrm{x}$.

Hay, M., Rastogi, V., Miklau, G. and Suciu, D. (2010) 'Boosting the accuracy of differentially private histograms through consistency', Proc. of the VLDB Endowment, Vol. 3, No. 1, pp.1021-1032, DOI: 10.14778/19 20841.1920970. 
Holohan, N., Leith, D.J. and Mason, O. (2017) 'Optimal differentially private mechanisms for randomised response', IEEE Transactions on Information Forensics and Security, Vol. 12, No. 11, pp.2726-2735, DOI: 10.1109/TIF S.2017. 2718487.

Karwa, V., Slavković, A.B. and Krivitsky, P. (2014) 'Differentially private exponential random graphs', Proc. of Int. Conference on Privacy in Statistical Databases, pp.143-155, DOI: 10.1007/978-3-319-11257-2_12.

Kasiviswanathan, S.P., Nissim, K., Raskhodnikova, S. and Smith, A. (2013) 'Analyzing graphs with node-differential privacy', Proc. of 10th Theory of Cryptography Conference, pp.457-476, DOI: 10.1007/978-3-642-36594-2_26.

Khandelwal, S. (2018) 'Facebook and Cambridge analytica what's happened so far', The Hacker News, 23 March [online] https://thehackernews.com/2018/03/ facebookcambridge-analytica.html.

Li, X-y., Yang, J. and Sun, Z-1. (2017) 'Differential privacy for edge weights in social networks', Security and Communication Networks, No. 2017, pp.1-10, DOI: 10.1155/ $2017 / 4267921$

Liu, K. and Terzi, E. (2008) 'Towards identity anonymization on graphs', Proc. of the 2008 ACM SIGMOD Int. Conference on Management of Data, pp.93-106, DOI: 10.1145/1376616. 1376629.

Macwan, K.R. and Patel, S.J. (2018) 'k-NMF anonymization in social network data publishing', The Computer J., Vol. 61, No. 4, pp.601-613, DOI: 10.1093/comjnl/bxy012.

Mittal, P., Papamanthou, C. and Song, D. (2013) 'Preserving link privacy in social network based systems', Proc. of 20th Annual Network and Distributed System Security Symposium, pp.1-11.
Nguyen, H.H., Imine, A. and Rusinowitch, M. (2014) 'A maximum variance approach for graph anonymization', Foundations and Practice of Security, Vol. 8930, pp.49-64, DOI: 10.1007/978-3-319-17040-4_4.

Nguyen, H.H., Imine, A. and Rusinowitch, M. (2015) 'Anonymizing social graphs via uncertainty semantics', Proc. of the 10th ACM Symposium on Information, Computer and Communications Security, pp.495-506, DOI: 10.1145/ 2714576.2714584

Qian, Q., Li, Z-x., Zhao, P-p. et al. (2018) 'Publishing graph node strength histogram with edge differential privacy', Proc. of Int. Conference on Database Systems for Advanced Applications, pp.75-91, DOI: 10.1007/978-3-319-91458-9_5.

Van den Hout, A. and Van der Heijden, P.G.M. (2002) 'Randomized response, statistical disclosure control and misclassificatio: a review', Int. Statistical Review, Vol. 70, No. 2, pp.269-288, DOI: 10.1111/j.1751-5823.2002.tb0 0363.x.

Ying, X-w., Pan, K., Wu, X-t. et al. (2009) 'Comparisons of randomization and $\mathrm{k}$-degree anonymization schemes for privacy preserving social network publishing', Proc. of the 3rd Workshop on Social Network Mining and Analysis, pp.1-10, DOI: 10.1145/1731011.1731021.

Yu, F-h., Chen, M-j., Yu, B-l. et al. (2018) 'Privacy preservation based on clustering perturbation algorithm for social network', Multimedia Tools \& Applications, Vol. 77, No. 9, pp.11241-11258, DOI: 10.1007/s11042-017-5502-3.

Zhou, B. and Pei, J. (2008) 'Preserving privacy in social networks against neighborhood attacks', Proc. of IEEE 24th Int. Conference on ICDE, pp.506-515, DOI: 10.1109/ICDE. 2008.4497459 